\title{
Factors Related to the Weight Control Practices of Korean Adults
}

\author{
Hyo Young Lee', Bo Kyung Hong ${ }^{2, *}$ \\ ${ }^{1}$ Department of Health Administration, Dongseo University, Busan; ${ }^{2}$ Department of Beauty, Kyungil University, Gyeongsan, Korea
}

Background: Maintenance of proper weight can help protect against chronic diseases. This study investigated the weight control practices of Korean adults and associated factors.

Methods: Secondary data from a nationwide cross-sectional survey of the fifth Korea National Health and Nutrition Examination Survey from 2016 to 2018 were analyzed. Subjects were aged 21 to 80 years ( $n=14,661)$. The influences of socio-demographic characteristics, health status, health behaviors, and perception of body image on weight control efforts were identified.

Results: Women (odds ratio [OR], 1.191; 95\% confidence interval [Cl], 1.723-2.210), city dwellers (OR, 1.229; $95 \% \mathrm{Cl}, 1.097-1.377$ ), those who had a middle school or higher level of education (OR, from 1.281 to 1.525 ), individuals who perceived themselves to be healthy or in fairly health (OR, from 1.103 to 1.257 ), those with high blood pressure (OR, 1.244; 95\% Cl, 1.104-1.401), normal or obese body mass index (BMl; OR from 2.579 to 4.430), non-smokers (OR, 1.373; $95 \% \mathrm{Cl}, 1.192-1.582)$, and those who drank alcohol $(\mathrm{OR}, 1.174 ; 95 \% \mathrm{Cl}, 1.057-$ 1.305) made more weight loss efforts than other individuals. Regardless of actual BMI, if someone perceived themselves to be obese or normal weight (OR, from 4.324 to 10.884), they made more effort to reduce their weight than those individuals who perceived themselves to be underweight.

Conclusion: Weight control is a positive behavior for people with high BMI. However, those individuals with a normal weight who believe they are obese may require further education. It is also necessary to monitor and address the lack of weight control efforts in rural residents and that there is a need for weight control counseling and support for people with high blood pressure and diabetes. Various related causes should be considered and community-wide efforts be made to support people who need weight control.

Key words: Body weight, Weight loss, Health behavior, Related factor
Received April 3, 2021

Reviewed October 25, 2021

Accepted November 11, 2021

*Corresponding author Bo Kyung Hong

(iD) https://orcid.org/0000-0001-9811-9541

Department of Beauty, Kyungil University, 50 Gamasil-gil, Hayang-eup, Gyeongsan 38428, Korea Tel: +82-53-600-5884

Fax: +82-53-600-5899

E-mail:karma0616@naver.com

\section{INTRODUCTION}

Obesity is a global public health problem, and the incidences of diseases caused by obesity are rapidly increasing. Maintenance of a proper weight can protect against chronic diseases such as obesity, hypertension, diabetes, cardiovascular disease, coronary heart disease, and cancer. Such weight control is essential through healthy behaviors such as exercise and proper diet. ${ }^{1}$ Additionally, women prefer to have slim bodies due to the influence of media and often desire to lose weight even if their body weight is normal. ${ }^{2}$ Weight control generally means trying to lose or maintain weight intentionally. ${ }^{3}$ Chronic diseases such as cardiovascular disease, hypertension, and coronary heart disease are also factors that affect weight control behaviors. ${ }^{4}$ Furthermore, an increase in the incidence of diabetes and obesity has resulted in great public interest in weight control, diet behaviors, and exercise. Weight control is also related to health-related quality of life. ${ }^{5}$ However, the desire for weight control may also reflect psychosocial factors. ${ }^{6}$

Many studies have examined factors that affect weight control. However, the populations that have been studied have been highly 
specific, such as female high school students, female college students, and adolescent women. ${ }^{3,7-10}$ Neumark-Sztainer et al. ${ }^{3}$ stated that weight control behaviors among adolescents were of two types: healthy weight control by exercising, and unhealthy weight control achieved by limiting the number of meals, vomiting, or adopting a one-food diet. Oh et al. ${ }^{8}$ reported that factors common to adolescents exhibiting healthy weight control behaviors were female sex, higher economic status, a mother with a university education, and normal weight. A study on girls showed that negative attitudes toward the body were triggers for attempting weight loss. ${ }^{7}$ In a study by Yang et al. ${ }^{10}$ on girls, obesity, self-esteem, and body esteem were relevant to unhealthy weight loss. According to Kim ${ }^{9}$, perceived level of health, subjective body perceptions, drinking, leisure activities, stress, and depressive experiences influenced weight loss in young Korean women.

Here, rather than focusing on any particular population, we investigated factors related to weight control for an entire group of adults. Specifically, this study aimed to determine how socio-demographic characteristics, health conditions, health practices, and weight control behaviors influence weight control in adults by utilizing the most recent data from a health survey of a representative sample of the adult population of Korea.

\section{METHODS}

\section{Study design \& participants}

This was a secondary analysis of data from the nationwide crosssectional survey of the fifth Korea National Health and Nutrition Examination Survey conducted from 2016 to 2018 . The survey received Institutional Review Board approval (IRB No. 2018-01-03P-A) and written informed consent was obtained from all respondents before data collection. ${ }^{11}$ Participants were sampled using a complex, stratified, multistage probability method, and the response rate was $76.6 \% .{ }^{11}$ Among a total of 14,661 participant records, subjects (men, 6,177; women, 8,484) aged 21 to 80 years with no main missing variables such as experience with weight control, socio-economic variables, health status, and health behaviors and cognition, were considered in this study. Permission to use raw data was obtained from the Korea Center for Disease Control, and data were downloaded directly from the website (Fig. 1).

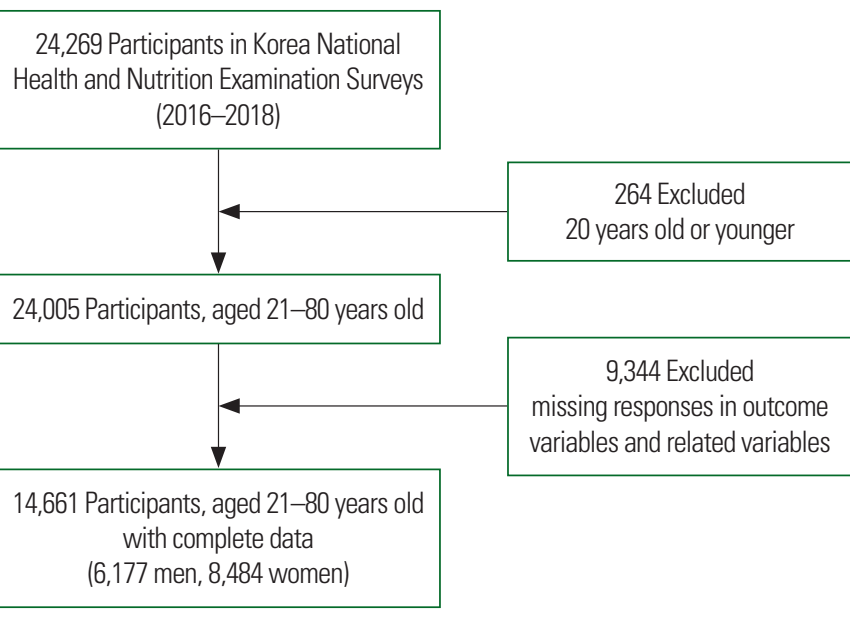

Figure 1. Study participants.

\section{Measurements}

\section{Dependent variable: weight control}

Any experience with weight control was assessed by asking, "Have you ever controlled your body weight in the past year?" Responses were assigned to one of four categories: efforts to reduce weight, maintain weight, gain weight, or no attempts to modify weight.

\section{Independent variables}

Socio-demographic status (sex, age group, household income level, employment status, marital status, residential area, educational attainment), health status (perceived subjective health status, hypertension, diabetes, depression, body mass index [BMI]), and health behaviors and perceptions (current alcohol drinking, current smoking, sleep hours, subjective perception of body shape, stress, sitting hours, amount and types of food eaten) were included to explore factors associated with weight control efforts.

\section{Socio-demographic characteristics}

Socio-demographic characteristics consisted of sex (men or women), age group ( $21-30,31-40,41-50,51-60,61-70,71-80$ years) household income level (lower, lower intermediate, upper intermediate, or upper), employment (yes or no), marital status (married or unmarried), residential area (urban or rural), and educational attainment (no education or elementary school graduate, middle school graduate, high school graduate, college graduate or higher). 


\section{Health status}

Health status included perceived health status, hypertension, diabetes, and depression (disease diagnosed by a doctor). Participants were asked, "In general, what do you think of your health?" with five categories of responses (excellent, good, fair, poor, or very poor) to assess perceived general health status. Responses were divided into healthy (excellent/good), fair, and not healthy (poor/ very poor). Regarding diseases, participants were asked whether they had hypertension, diabetes, and/or depression (based on doctors' diagnoses). Their status was coded as "do not have" or "have". Four weight categories were recognized based on BMI: underweight $\left(\mathrm{BMI}<18.5 \mathrm{~kg} / \mathrm{m}^{2}\right)$, normal weight $\left(18.5 \mathrm{~kg} / \mathrm{m}^{2} \leq \mathrm{BMI}\right.$ $\left.<25 \mathrm{~kg} / \mathrm{m}^{2}\right)$, overweight $\left(25 \mathrm{~kg} / \mathrm{m}^{2} \leq \mathrm{BMI}<30 \mathrm{~kg} / \mathrm{m}^{2}\right)$, and obese $\left(\mathrm{BMI} \geq 30 \mathrm{~kg} / \mathrm{m}^{2}\right)$. Anthropometric measurements of height and weight were published in detail previously (Ministry of Health and Welfare, and Korea Center for Disease Control and Prevention, 2018).

\section{Health behaviors and cognition}

Health behaviors and cognition consisted of current alcohol drinking (yes or no), current smoking (yes or no), sleep hours ( $\geq 7$ sleeping hours versus $<7$ sleeping hours), sitting hours ( $<7$ hours, 7-12 hours, and 12 hours and over), subjective cognition of body shape (normal, obese, and thin), stress (nearly none, a little, a lot), and type and amount of food eaten. Participants were asked, "How many hours on average do you sit in a day?" and their responses were divided into three categories. Participants were also asked, "Which statement most closely describes the type and amount of food you eat at home?" and responses offered were "sufficient and varied," "sufficient but not varied," and "insufficient." As for stress, participants were asked, "How much stress do you feel on an ordinary day?" with responses of nearly none, a little, or a lot.

\section{Data analysis}

Data were analyzed using IBM SPSS version 23.0 (IBM Corp., Armonk, NY, USA). Pooled sampling weights were applied to all data to estimate representative statistics for Korea's adult population. Results are presented as weighted percentages (with standard errors) and odds ratios (ORs) with 95\% confidence intervals (CIs). The chi-square test was used to determine the significance of dif- ferences in the distribution of participants' characteristics and differences in weight control experience prevalence according to participants' characteristics. Multiple logistic regression analysis was performed to explore factors associated with weight control experiences after controlling for related variables (socio-demographic variables, health status variables, and health behaviors \& cognition variables). This analysis was conducted separately to identify factors related to each weight control practice, namely "efforts to reduce weight," "efforts to maintain weight," or "efforts to gain weight," compared to those who had never made any effort to control their weight. All independent variables in this study were included in the logistic regression model. Variables with a $P>0.05$, especially depression and sleep hours, were removed from the full model.

\section{RESULTS}

\section{General characteristics of the study population}

More than half of the study subjects were female (52.0\%) and $20.9 \%$ of participants were 41 to 50 years old. A total of $64.5 \%$ individuals were working professionals, $79.2 \%$ were married, and $85.1 \%$ were urban residents. Overall, $42.9 \%$ had the highest education level of college graduation or higher. The subjective health status of fair was most common at 52.3\%, 19.9\% had hypertension (diagnosed by a doctor), $7.6 \%$ had diabetes, $4.2 \%$ had depression, and $60.9 \%$ had a normal BMI. At the time of this study, $76.0 \%$ drank alcohol, 19.9\% smoked, and 52.3\% slept less than 7 hours a day. With regard to weight control over the past year, $41.4 \%$ had made efforts to reduce weight, and $34.5 \%$ had never tried to control their weight. Among participants, $45.0 \%$ considered themselves obese. Furthermore, $57.4 \%$ experienced a little stress on a daily basis while $15 \%$ did not report feeling stressed. Most participants sat for between 7 and 12 hours a day $(40.1 \%)$, and $56.6 \%$ consumed sufficient amounts of varied foods (Table 1).

\section{Socio-demographic and health status and health behaviors}

Among women, $47.0 \%$ reported efforts to reduce weight while most men (38.5\%) never tried to control their weight. Furthermore, most people in all age groups made efforts to reduce weight. Efforts to maintain weight increased with age, except for those aged 
Table 1. Characteristics of the study participants

\begin{tabular}{|c|c|}
\hline Characteristics & No. $(\%)$ \\
\hline \multicolumn{2}{|l|}{ Socio-demographic status } \\
\hline \multicolumn{2}{|l|}{ Sex } \\
\hline Male & $6,177(48.0)$ \\
\hline Female & $8,484(52.0)$ \\
\hline \multicolumn{2}{|l|}{ Age group (yr) } \\
\hline $21-30$ & $1,629(16.8)$ \\
\hline $31-40$ & $2,483(19.1)$ \\
\hline $41-50$ & $2,754(20.9)$ \\
\hline $51-60$ & 2,847 (19.7) \\
\hline $61-70$ & $2,629(13.2)$ \\
\hline $71-80$ & $2,319(10.2)$ \\
\hline \multicolumn{2}{|l|}{ Household income level } \\
\hline Low level & $3,583(25.2)$ \\
\hline Lower-middle level & $3,672(24.9)$ \\
\hline Upper-middle level & $3,689(25.1)$ \\
\hline High level & $3,717(24.9)$ \\
\hline \multicolumn{2}{|l|}{ Employed } \\
\hline No & $5,788(35.5)$ \\
\hline Yes & $8,873(64.5)$ \\
\hline \multicolumn{2}{|l|}{ Marital status } \\
\hline Married & $12,517(79.2)$ \\
\hline Unmarried & 2,144 (20.8) \\
\hline \multicolumn{2}{|l|}{ Residential area } \\
\hline Urban & $11,913(85.1)$ \\
\hline Rural & $2,748(14.9)$ \\
\hline \multicolumn{2}{|l|}{ Education Attainment } \\
\hline No education or elementary school graduate & $3,050(14.9)$ \\
\hline Middle school & 1,508 (8.8) \\
\hline High school & $4,506(33.4)$ \\
\hline College graduate or higher & $5,597(42.9)$ \\
\hline \multicolumn{2}{|l|}{ Health status } \\
\hline \multicolumn{2}{|l|}{ Perceived subjective health status } \\
\hline Not healthy & 2,913 (17.8) \\
\hline Fair & $7,634(52.3)$ \\
\hline Healthy & $4,114(29.9)$ \\
\hline \multicolumn{2}{|l|}{ Hypertension } \\
\hline Do not have & 10,999 (80.1) \\
\hline Have & $3,662(19.9)$ \\
\hline \multicolumn{2}{|l|}{ Diabetes } \\
\hline Do not have & $13,227(92.4)$ \\
\hline Have & $1,434(7.6)$ \\
\hline \multicolumn{2}{|l|}{ Depression } \\
\hline Do not have & 13,982 (95.8) \\
\hline Have & $679(4.2)$ \\
\hline \multicolumn{2}{|l|}{ Body mass index } \\
\hline Underweight & $542(3.9)$ \\
\hline Normal & $9,012(60.9)$ \\
\hline Obese & $5,107(35.2)$ \\
\hline
\end{tabular}

Table 1. Continued

\begin{tabular}{|c|c|}
\hline Characteristics & №. $(\%)$ \\
\hline \multicolumn{2}{|l|}{ Health behaviors \& cognition } \\
\hline \multicolumn{2}{|l|}{ Current alcohol drinking } \\
\hline No & $4,134(24.0)$ \\
\hline Yes & $10,527(76.0)$ \\
\hline \multicolumn{2}{|l|}{ Current smoking } \\
\hline No & $12,217(80.1)$ \\
\hline Yes (ref.) & $2,444(19.9)$ \\
\hline \multicolumn{2}{|l|}{ Sleep hours } \\
\hline Less than 7 hours a night & $7,622(52.3)$ \\
\hline 7 or more hours a night & $7,039(47.7)$ \\
\hline \multicolumn{2}{|l|}{ Weight control (per year) } \\
\hline Efforts to reduce weight & $5,906(41.4)$ \\
\hline Efforts to maintain weight & $2,759(18.8)$ \\
\hline Efforts to gain weight & $760(5.3)$ \\
\hline Never tried & $5,236(34.5)$ \\
\hline \multicolumn{2}{|c|}{ Subjective perception of body shape } \\
\hline Underweight & $2,266(15.4)$ \\
\hline Normal & $5,921(36.9)$ \\
\hline Obese & $6,474(45.0)$ \\
\hline \multicolumn{2}{|l|}{ Stress } \\
\hline Nearly none & $2,523(15.0)$ \\
\hline A little & $8,287(57.4)$ \\
\hline A lot & $3,851(27.5)$ \\
\hline \multicolumn{2}{|l|}{ Sitting hours (per day) } \\
\hline Less than 7 hours & $5,593(37.2)$ \\
\hline Between 7 and 12 hours & $5,880(40.1)$ \\
\hline 12 or more hours & $3,188(22.7)$ \\
\hline \multicolumn{2}{|l|}{ Amount and type of food eaten } \\
\hline Sufficient \& varied & $8,069(56.6)$ \\
\hline Sufficient but not varied & $6,167(40.8)$ \\
\hline Insufficient & $425(2.6)$ \\
\hline
\end{tabular}

71-80 years. However, the higher the income level, the more significant the efforts to reduce weight with a decrease in the number of individuals who had never tried to control weight. Those individuals who had never tried to control weight were predominantly from rural areas. Moreover, the lower the level of education, the more likely an individual was to have never tried to control weight. In other words, people with higher levels of education had more weight-control practices than those with no or lower levels of education (Table 2).

With respect to subjective health, about $68 \%$ of the people who judged themselves to be healthy tried to control their weight. People with hypertension and diabetes made less weight control efforts than those who did not. According to data on BMI and subjective 
Table 2. Socio-demographic factors, health status, and health behaviors according to weight control group

\begin{tabular}{|c|c|c|c|c|c|c|c|c|c|}
\hline \multirow[t]{2}{*}{ Variable } & \multicolumn{2}{|c|}{$\begin{array}{l}\text { Effort to reduce weight } \\
\qquad(n=5,906)\end{array}$} & \multicolumn{2}{|c|}{$\begin{array}{l}\text { Effort to maintain weight } \\
\qquad(\mathrm{n}=2,759)\end{array}$} & \multicolumn{2}{|c|}{$\begin{array}{l}\text { Effort to gain weight } \\
\qquad(\mathrm{n}=760)\end{array}$} & \multicolumn{2}{|c|}{$\begin{array}{l}\text { Never tried } \\
(n=5,236)\end{array}$} & \multirow[t]{2}{*}{$P$} \\
\hline & $\%$ & SE & $\%$ & SE & $\%$ & SE & $\%$ & SE & \\
\hline Socio-demographic status & & & & & & & & & \\
\hline Sex & & & & & & & & & $<0.001$ \\
\hline Male & 35.4 & 0.7 & 18.5 & 0.6 & 7.6 & 0.4 & 38.5 & 0.8 & \\
\hline Female & 47.0 & 0.7 & 19.1 & 0.6 & 3.1 & 0.2 & 30.8 & 0.6 & \\
\hline $31-40$ & 46.4 & 1.1 & 18.0 & 0.9 & 4.1 & 0.5 & 31.5 & 1.1 & \\
\hline $41-50$ & 45.9 & 1.1 & 19.3 & 0.8 & 4.1 & 0.5 & 30.8 & 1.0 & \\
\hline $51-60$ & 41.7 & 1.1 & 22.3 & 0.9 & 4.6 & 0.5 & 31.4 & 1.0 & \\
\hline $61-70$ & 36.9 & 1.1 & 20.8 & 1.0 & 5.7 & 0.5 & 36.7 & 1.2 & \\
\hline $71-80$ & 21.1 & 1.0 & 13.4 & 0.9 & 5.3 & 0.6 & 58.0 & 1.3 & \\
\hline Upper-middle level & 43.3 & 1.0 & 18.2 & 0.7 & 5.1 & 0.4 & 33.4 & 0.9 & \\
\hline High level & 41.7 & 1.1 & 22.7 & 0.8 & 5.0 & 0.4 & 30.6 & 0.9 & \\
\hline Employed & & & & & & & & & 0.320 \\
\hline No & 40.0 & 0.8 & 18.4 & 0.6 & 5.7 & 0.4 & 35.9 & 0.8 & \\
\hline Yes & 42.2 & 0.6 & 19.0 & 0.5 & 5.0 & 0.3 & 33.7 & 0.6 & \\
\hline Marital status & & & & & & & & & $<0.001$ \\
\hline Married & 40.8 & 0.5 & 19.3 & 0.4 & 4.7 & 0.2 & 35.2 & 0.5 & \\
\hline Unmarried & 43.9 & 1.2 & 16.7 & 0.9 & 7.4 & 0.7 & 32.0 & 1.2 & \\
\hline Residential area & & & & & & & & & $<0.001$ \\
\hline Urban & 42.5 & 0.5 & 19.2 & 0.4 & 5.1 & 0.3 & 33.3 & 0.5 & \\
\hline Rural & 35.1 & 1.3 & 16.7 & 1.0 & 6.5 & 0.6 & 41.7 & 1.4 & \\
\hline Perceived subjective health status & & & & & & & & & $<0.001$ \\
\hline Not healthy & 39.2 & 1.1 & 14.9 & 0.8 & 7.4 & 0.6 & 38.5 & 1.1 & \\
\hline Fair & 41.4 & 0.7 & 19.0 & 0.5 & 4.8 & 0.3 & 34.7 & 0.6 & \\
\hline Healthy & 42.7 & 1.0 & 20.8 & 0.7 & 4.8 & 0.4 & 31.7 & 0.9 & \\
\hline Hypertension & & & & & & & & & $<0.001$ \\
\hline Do not have & 41.8 & 0.6 & 19.5 & 0.4 & 5.5 & 0.3 & 33.2 & 0.6 & \\
\hline Have & 40.1 & 1.0 & 15.8 & 0.7 & 4.3 & 0.4 & 39.8 & 1.0 & \\
\hline Diabetes & & & & & & & & & $<0.001$ \\
\hline Do not have & 41.8 & 0.5 & 19.0 & 0.4 & 5.3 & 0.2 & 33.9 & 0.5 & \\
\hline Have & 36.8 & 1.5 & 16.3 & 1.2 & 5.2 & 0.7 & 41.7 & 1.6 & \\
\hline Depression & & & & & & & & & 0.460 \\
\hline Do not have & 41.3 & 0.5 & 18.9 & 0.4 & 5.2 & 0.2 & 34.6 & 0.5 & \\
\hline Have & 44.0 & 2.2 & 16.9 & 1.8 & 6.0 & 1.0 & 33.1 & 2.1 & \\
\hline Body mass index & & & & & & & & & $<0.001$ \\
\hline Underweight & 7.8 & 1.2 & 14.5 & 1.7 & 30.8 & 2.5 & 46.9 & 2.5 & \\
\hline Normal & 33.4 & 0.6 & 22.6 & 0.5 & 6.4 & 0.3 & 37.6 & 0.6 & \\
\hline Obese & 59.0 & 0.8 & 12.8 & 0.6 & 0.4 & 0.1 & 27.8 & 0.7 & \\
\hline
\end{tabular}


Table 2. Continued

\begin{tabular}{|c|c|c|c|c|c|c|c|c|c|}
\hline \multirow[t]{2}{*}{ Variable } & \multicolumn{2}{|c|}{$\begin{array}{l}\text { Effort to reduce weight } \\
\qquad(n=5,906)\end{array}$} & \multicolumn{2}{|c|}{$\begin{array}{l}\text { Effort to maintain weight } \\
\qquad(\mathrm{n}=2,759)\end{array}$} & \multicolumn{2}{|c|}{$\begin{array}{l}\text { Effort to gain weight } \\
\qquad(n=760)\end{array}$} & \multicolumn{2}{|c|}{$\begin{array}{l}\text { Never tried } \\
(n=5,236)\end{array}$} & \multirow[t]{2}{*}{$P$} \\
\hline & $\%$ & SE & $\%$ & SE & $\%$ & SE & $\%$ & SE & \\
\hline \multicolumn{10}{|l|}{ Health behavior \& cognition } \\
\hline Current alcohol drinker & & & & & & & & & $<0.001$ \\
\hline No & 37.5 & 0.9 & 17.2 & 0.7 & 5.5 & 0.4 & 39.9 & 0.9 & \\
\hline Yes & 42.7 & 0.6 & 19.3 & 0.4 & 5.2 & 0.3 & 32.8 & 0.6 & \\
\hline Current smoker & & & & & & & & & $<0.001$ \\
\hline No & 43.4 & 0.5 & 19.5 & 0.4 & 4.5 & 0.2 & 32.5 & 0.5 & \\
\hline Yes (ref) & 33.5 & 1.2 & 15.8 & 0.8 & 8.3 & 0.7 & 42.5 & 1.2 & \\
\hline Sleep hours & & & & & & & & & 0.051 \\
\hline Less than 7 hours & 40.3 & 0.7 & 19.0 & 0.5 & 5.6 & 0.3 & 35.1 & 0.7 & \\
\hline 7 or more hours & 42.7 & 0.7 & 18.6 & 0.5 & 4.9 & 0.3 & 33.9 & 0.7 & \\
\hline Subjective perception of body shape & & & & & & & & & $<0.001$ \\
\hline Underweight & 7.5 & 0.7 & 16.6 & 0.9 & 25.9 & 1.1 & 50.0 & 1.2 & \\
\hline Normal & 31.5 & 0.8 & 26.2 & 0.7 & 2.9 & 0.3 & 39.5 & 0.8 & \\
\hline Obese & 61.8 & 0.7 & 13.1 & 0.5 & 0.3 & 0.1 & 24.8 & 0.6 & \\
\hline Stress & & & & & & & & & $<0.001$ \\
\hline Nearly none & 36.0 & 1.2 & 17.2 & 0.9 & 4.2 & 0.4 & 42.6 & 1.2 & \\
\hline A little & 41.7 & 0.7 & 20.5 & 0.5 & 5.2 & 0.3 & 32.6 & 0.7 & \\
\hline A lot & 43.7 & 0.9 & 16.2 & 0.6 & 6.0 & 0.5 & 34.1 & 0.9 & \\
\hline Sitting hours (per day) & & & & & & & & & 0.001 \\
\hline Less than 7 hours & 39.6 & 0.8 & 20.1 & 0.7 & 5.3 & 0.4 & 35.0 & 0.8 & \\
\hline Between 7 and 12 hours & 42.9 & 0.8 & 18.9 & 0.6 & 5.4 & 0.4 & 32.8 & 0.8 & \\
\hline 12 or more hours & 41.9 & 1.1 & 16.4 & 0.8 & 5.0 & 0.5 & 36.8 & 1.0 & \\
\hline Amount and type of food eaten & & & & & & & & & $<0.001$ \\
\hline Sufficient \& varied & 43.0 & 0.7 & 19.5 & 0.5 & 5.0 & 0.3 & 32.5 & 0.7 & \\
\hline Sufficient but not varied & 39.6 & 0.8 & 18.2 & 0.6 & 5.6 & 0.4 & 36.6 & 0.8 & \\
\hline Insufficient & 36.3 & 2.8 & 11.4 & 1.5 & 5.9 & 1.2 & 46.5 & 2.8 & \\
\hline
\end{tabular}

SE, standard error.

body perception, obese people made the most weight control efforts (Table 2). Furthermore, people who drank, did not smoke, and were more stressed, as well as people with a lot of sedentary time engaged in more weight control efforts. With respect to the dietary intake environment, those who reported eating sufficient and varied food groups controlled their weight more than those individuals who ate sufficient but not varied food or insufficient food (Table 2).

\section{Factors related to efforts to reduce body weight}

Women made more effort to reduce body weight than men (1.191-fold more; 95\% CI, 1.723-2.210). In addition, individuals older than 51 years made significantly less effort to reduce weight than people in their 20s. People living in cities were more likely to report attempts to reduce weight than those living in rural areas. In addition, individuals who had graduated middle school (OR, 1.281 ; $95 \%$ CI, $1.079-1.521$ ) or higher (OR from 1.338 to 1.525 ) made more weight reduction efforts than individuals who had no education or had only graduated elementary school (Table 3).

Perceived subjective health status, hypertension, and BMI were significantly associated with weight reduction behaviors, and obese individuals made 4.430-fold the effort to reduce their weight than underweight individuals (95\% CI, 3.078-6.377). Furthermore, alcohol drinkers made a 1.174-fold greater effort to reduce their weight than other subjects, and non-smokers engaged more in weight reduction than smokers. Regardless of actual BMI, if someone perceived themselves to be obese, they made more weight reducing efforts (10.884-fold greater than the other study participants) (Table 3 ). 
Table 3. Factors related to efforts to reduce weight

\begin{tabular}{|c|c|c|c|}
\hline Factor & Odds ratio & $95 \% \mathrm{Cl}$ & $P$ \\
\hline \multicolumn{4}{|l|}{ Socio-demographic status } \\
\hline \multicolumn{4}{|l|}{ Sex } \\
\hline Female* & 1.191 & $1.723-2.210$ & 0.000 \\
\hline Male (ref) & 1.000 & & \\
\hline \multicolumn{4}{|l|}{ Age group (yr) } \\
\hline $31-40$ & 0.886 & $0.736-1.535$ & 0.000 \\
\hline $41-50$ & 0.829 & $0.736-1.066$ & 0.000 \\
\hline $51-60^{*}$ & 0.772 & $0.622-0.958$ & 0.000 \\
\hline $61-70^{*}$ & 0.679 & $0.540-0.853$ & 0.000 \\
\hline $71-80^{*}$ & 0.345 & $0.262-0.454$ & 0.000 \\
\hline 21-30 (ref) & 1.000 & & \\
\hline \multicolumn{4}{|l|}{ Household income level } \\
\hline Lower-middle level & 1.040 & $0.915-1.183$ & 0.528 \\
\hline Upper-middle level & 1.134 & $0.977-1.288$ & 0.988 \\
\hline High level & 1.069 & $0.930-1.228$ & 0.201 \\
\hline Low level (ref) & 1.000 & & \\
\hline \multicolumn{4}{|l|}{ Employed } \\
\hline Yes & 1.046 & $0.946-1.157$ & 0.488 \\
\hline No (ref) & 1.000 & & \\
\hline \multicolumn{4}{|l|}{ Marital status } \\
\hline Unmarried & 1.102 & $0.928-1.308$ & 0.255 \\
\hline Married (ref) & 1.000 & & \\
\hline \multicolumn{4}{|l|}{ Residential area } \\
\hline Urban* & 1.229 & $1.097-1.377$ & 0.000 \\
\hline Rural (ref) & 1.000 & & \\
\hline \multicolumn{4}{|l|}{ Education Attainment } \\
\hline Middle school* & 1.281 & $1.079-1.521$ & 0.000 \\
\hline High school* & 1.338 & $1.153-1.552$ & 0.041 \\
\hline College graduate or higher* & 1.525 & $1.300-1.789$ & 0.021 \\
\hline $\begin{array}{l}\text { No education or elementary school } \\
\text { graduate (ref) }\end{array}$ & 1.000 & & \\
\hline \multicolumn{4}{|l|}{ Health status } \\
\hline \multicolumn{4}{|l|}{ Perceived subjective health status } \\
\hline Fair* & 1.103 & $0.980-0.242$ & 0.002 \\
\hline Healthy* & 1.257 & $1.090-1.449$ & 0.012 \\
\hline Not healthy (ref) & 1.000 & & \\
\hline \multicolumn{4}{|l|}{ Hypertension } \\
\hline Have $^{*}$ & 1.244 & $1.104-1.401$ & 0.001 \\
\hline Do not have (ref) & 1.000 & & \\
\hline \multicolumn{4}{|l|}{ Diabetes } \\
\hline Have & 1.071 & $0.912-1.258$ & 0.452 \\
\hline Do not have (ref) & 1.000 & & \\
\hline \multicolumn{4}{|l|}{ Body mass index } \\
\hline Normal $^{*}$ & 2.579 & $1.834-3.628$ & 0.000 \\
\hline Obese ${ }^{*}$ & 4.430 & $3.078-6.377$ & 0.000 \\
\hline Underweight (ref) & 1.000 & & \\
\hline
\end{tabular}

(Continued to the next)
Table 3. Continued

\begin{tabular}{|c|c|c|c|}
\hline Factor & Odds ratio & $95 \% \mathrm{Cl}$ & $P$ \\
\hline \multicolumn{4}{|l|}{ Health behavior \& cognition } \\
\hline \multicolumn{4}{|l|}{ Current alcohol drinker } \\
\hline Yes $^{*}$ & 1.174 & $1.057-1.305$ & 0.010 \\
\hline No (ref) & 1.000 & & \\
\hline \multicolumn{4}{|l|}{ Current smoker } \\
\hline $\mathrm{No}^{*}$ & 1.373 & $1.192-1.582$ & 0.000 \\
\hline Yes (ref) & 1.000 & & \\
\hline \multicolumn{4}{|c|}{ Subjective perception of body shape } \\
\hline Normal $^{*}$ & 4.324 & $3.459-5.183$ & 0.000 \\
\hline Obese $^{*}$ & 10.884 & $8.776-13.499$ & 0.000 \\
\hline Underweight (ref) & 1.000 & & \\
\hline \multicolumn{4}{|l|}{ Stress } \\
\hline Nearly none & 0.883 & $0.765-1.019$ & 0.178 \\
\hline A little & 0.980 & $0.889-1.082$ & 0.694 \\
\hline A lot (ref) & 1.000 & & \\
\hline \multicolumn{4}{|l|}{ Sitting hour (per day) } \\
\hline Fewer than 7 hours & 1.028 & $0.913-1.158$ & 0.852 \\
\hline Between 7 and 12 hours & 1.113 & $0.985-1.257$ & 0.113 \\
\hline 12 or more hours (ref) & 1.000 & & \\
\hline \multicolumn{4}{|c|}{ Amount and type of food eaten } \\
\hline Sufficient but not varied & 0.913 & $0.892-1.006$ & 0.700 \\
\hline Insufficient & 1.030 & $0.762-1.390$ & 0.317 \\
\hline Sufficient and varied (ref) & 1.000 & & \\
\hline
\end{tabular}

\section{Factors related to any efforts to maintain body weight}

Individuals between the ages of 61 and 80 made significantly more effort to maintain their weight than individuals in their 20s. In addition, people who reported a high household income level made more effort to maintain body weight than people who reported a low household income level. Individuals who had graduated middle school made 1.459-fold more effort to maintain their weight than individuals with other levels of education. No other health status factors were associated with efforts to control weight. If someone perceived themselves to be obese, they made less effort to maintain their weight than those who perceived themselves to be underweight. People who experienced a little bit of stress reported more weight maintaining practices than those who experienced a lot of stress (OR, 1.161 times: 95\% CI, 1.033-1.305). People who reported insufficient food appeared to have less weight maintaining behaviors (OR, 0.668; 95\% CI, 0.489-0.913) compared to those who reported sufficient food (Table 4). 
Table 4. Factors related to efforts for maintaining weight

\begin{tabular}{|c|c|c|c|}
\hline Factor & Odds ratio & $95 \% \mathrm{Cl}$ & $P$ \\
\hline \multicolumn{4}{|l|}{ Socio-demographic status } \\
\hline \multicolumn{4}{|l|}{ Sex } \\
\hline Female & 0.981 & $0.870-1.107$ & 0.084 \\
\hline Male (ref) & 1.000 & & \\
\hline \multicolumn{4}{|l|}{ Age group (yr) } \\
\hline $31-40$ & 1.066 & $0.844-1.346$ & 0.843 \\
\hline $41-50$ & 1.130 & $0.889-1.436$ & 0.840 \\
\hline $51-60$ & 1.469 & $1.134-1.903$ & 0.475 \\
\hline $61-70^{*}$ & 1.570 & $1.191-2.068$ & 0.002 \\
\hline $71-80^{*}$ & 1.070 & $0.785-1.458$ & 0.000 \\
\hline 21-30 (ref) & 1.000 & & \\
\hline \multicolumn{4}{|l|}{ Household income level } \\
\hline Lower-middle level & 1.049 & $0.907-1.213$ & 0.000 \\
\hline Upper-middle level & 1.033 & $0.900-1.086$ & 0.005 \\
\hline High level $^{*}$ & 1.274 & $1.105-1.469$ & 0.001 \\
\hline Low level (ref) & 1.000 & & \\
\hline \multicolumn{4}{|l|}{ Employed } \\
\hline Yes & 0.951 & $0.851-1.062$ & 0.307 \\
\hline No (ref) & 1.000 & & \\
\hline \multicolumn{4}{|l|}{ Marital status } \\
\hline Unmarried & 0.926 & $0.758-1.132$ & 0.474 \\
\hline Married (ref) & 1.000 & & \\
\hline \multicolumn{4}{|l|}{ Residential area } \\
\hline Urban & 1.079 & $0.921-1.263$ & 0.402 \\
\hline Rural (ref) & 1.000 & & \\
\hline \multicolumn{4}{|l|}{ Education attainment } \\
\hline Middle school* & 1.459 & $1.195-1.783$ & 0.000 \\
\hline High school & 1.698 & $1.390-2.073$ & 0.165 \\
\hline College graduate or higher & 1.671 & $1.359-2.056$ & 0.878 \\
\hline $\begin{array}{l}\text { No education or elementary school } \\
\text { graduate (ref) }\end{array}$ & 1.000 & & \\
\hline \multicolumn{4}{|l|}{ Health status } \\
\hline \multicolumn{4}{|l|}{ Perceived subjective health status } \\
\hline Fair & 1.055 & $0.908-1.227$ & 0.625 \\
\hline Healthy & 1.035 & $0.878-1.221$ & 0.836 \\
\hline Not healthy (ref) & 1.000 & & \\
\hline \multicolumn{4}{|l|}{ Hypertension } \\
\hline Have & 0.874 & $0.763-1.002$ & 0.095 \\
\hline Do not have (ref) & 1.000 & & \\
\hline \multicolumn{4}{|l|}{ Diabetes } \\
\hline Have & 0.997 & $0.822-1.208$ & 0.931 \\
\hline Do not have (ref) & 1.000 & & \\
\hline \multicolumn{4}{|l|}{ Body mass index } \\
\hline Normal & 1.285 & $0.975-1.695$ & 0.825 \\
\hline Obese $^{*}$ & 0.970 & $0.710-1.327$ & 0.000 \\
\hline Underweight (ref) & 1.000 & & \\
\hline
\end{tabular}

(Continued to the next)
Table 4. Continued

\begin{tabular}{|c|c|c|c|}
\hline Factor & Odds ratio & $95 \% \mathrm{Cl}$ & $P$ \\
\hline \multicolumn{4}{|l|}{ Health behavior \& cognition } \\
\hline \multicolumn{4}{|l|}{ Current alcohol drinker } \\
\hline Yes & 1.137 & $1.005-1.285$ & 0.086 \\
\hline No (ref) & 1.000 & & \\
\hline \multicolumn{4}{|l|}{ Current smoker } \\
\hline $\mathrm{No}^{*}$ & 1.241 & $1.066-1.446$ & 0.005 \\
\hline Yes (ref) & 1.000 & & \\
\hline \multicolumn{4}{|c|}{ Subjective perception of body shape } \\
\hline Normal $^{*}$ & 1.636 & $1.389-1.928$ & 0.025 \\
\hline Obese & 0.817 & $0.669-2.576$ & 0.999 \\
\hline Underweight (ref) & 1.000 & & \\
\hline \multicolumn{4}{|l|}{ Stress } \\
\hline Nearly none & 1.107 & $0.959-1.278$ & 0.557 \\
\hline Alittle* & 1.161 & $1.033-1.305$ & 0.011 \\
\hline A lot (ref) & 1.000 & & \\
\hline \multicolumn{4}{|l|}{ Sitting hours (per day) } \\
\hline Fewer than 7 ours & 1.107 & $0.959-1.278$ & 0.084 \\
\hline Between 7 and 12 hours & 1.060 & $1.033-1.305$ & 0.268 \\
\hline 12 hours or more (ref) & 1.000 & & \\
\hline \multicolumn{4}{|c|}{ Amount and type of food eaten } \\
\hline Sufficient but not varied & 1.001 & $0.895-1.119$ & 0.033 \\
\hline Insufficient* & 0.668 & $0.489-0.913$ & 0.031 \\
\hline Sufficient and varied (ref) & 1.000 & & \\
\hline
\end{tabular}

\section{Factors related to efforts to gain body weight}

Women made less effort to gain weight than men. People who were college graduates or higher made 0.674-fold less weight gaining efforts than those with lower levels of education. Only BMI among health status factors was statistically associated with weight gaining behaviors, and obese people made less weight gaining efforts than underweight individuals. Furthermore, non-smokers engaged in fewer weight gaining efforts than smokers. If someone perceived themselves to be obese or normal, they made less effort to gain weight. People who experienced little to no stress made less effort to gain weight than individuals who experienced a lot of stress (OR, 0.631 times: 95\% CI, 0.458-0.870) (Table 5).

\section{DISCUSSION}

This study identified factors related to the weight control behaviors of adult Koreans based on the analyses of the socio-demographic characteristics, health status, and health behaviors of Korean male 
Table 5. Factors related to efforts to gain weight

\begin{tabular}{|c|c|c|c|}
\hline Factor & Odds ratio & $95 \% \mathrm{Cl}$ & $P$ \\
\hline \multicolumn{4}{|l|}{ Socio-demographic status } \\
\hline \multicolumn{4}{|l|}{ Sex } \\
\hline Female* & 0.368 & $0.293-0.460$ & 0.000 \\
\hline Male (ref) & 1.000 & & \\
\hline \multicolumn{4}{|l|}{ Age group (yr) } \\
\hline $31-40$ & 0.844 & $0.513-1.389$ & 0.864 \\
\hline $41-50$ & 0.952 & $0.569-1.593$ & 0.624 \\
\hline $51-60$ & 0.840 & $0.499-1.414$ & 0.967 \\
\hline $61-70$ & 0.927 & $0.525-1.637$ & 0.561 \\
\hline $71-80$ & 0.916 & $0.504-1.667$ & 0.938 \\
\hline 21-30 (ref) & 1.000 & & \\
\hline \multicolumn{4}{|l|}{ Household income level } \\
\hline Lower-middle level & 0.758 & $0.563-1.019$ & 0.934 \\
\hline Upper-middle level & 0.953 & $0.729-1.246$ & 0.042 \\
\hline High level & 1.068 & $0.807-1.413$ & 0.430 \\
\hline Low level (ref) & 1.000 & & \\
\hline \multicolumn{4}{|l|}{ Employed } \\
\hline Yes & 0.863 & $0.682-1.093$ & 0.353 \\
\hline No (ref) & 1.000 & & \\
\hline \multicolumn{4}{|l|}{ Marital status } \\
\hline Unmarried & 1.165 & $0.761-1.783$ & 0.471 \\
\hline Married (ref) & 1.000 & & \\
\hline \multicolumn{4}{|l|}{ Residential area } \\
\hline Urban & 0.824 & $0.660-1.029$ & 0.088 \\
\hline Rural (ref) & 1.000 & & \\
\hline \multicolumn{4}{|l|}{ Education attainment } \\
\hline Middle school & 1.246 & $0.857-1.810$ & 0.018 \\
\hline High school & 1.120 & $0.821-1.526$ & 0.002 \\
\hline College graduate or higher* & 0.674 & $0.471-0.965$ & 0.000 \\
\hline $\begin{array}{l}\text { No education or elementary school } \\
\text { graduate (ref) }\end{array}$ & 1.000 & & \\
\hline \multicolumn{4}{|l|}{ Health status } \\
\hline \multicolumn{4}{|l|}{ Perceived subjective health status } \\
\hline Fair & 0.762 & $0.590-0.985$ & 0.202 \\
\hline Healthy & 0.782 & $0.571-1.072$ & 0.754 \\
\hline Not healthy (ref) & 1.000 & & \\
\hline \multicolumn{4}{|l|}{ Hypertension } \\
\hline Have & 0.936 & $0.721-1.215$ & 0.685 \\
\hline Do not have (ref) & 1.000 & & \\
\hline \multicolumn{4}{|l|}{ Diabetes } \\
\hline Have & 0.943 & & 0.768 \\
\hline Do not have (ref) & 1.000 & & \\
\hline \multicolumn{4}{|l|}{ Body mass index } \\
\hline Normal $^{*}$ & 0.422 & & 0.000 \\
\hline Obese $^{*}$ & 0.107 & & 0.000 \\
\hline Underweight (ref) & 1.000 & & \\
\hline
\end{tabular}

(Continued to the next)
Table 5. Continued

\begin{tabular}{|c|c|c|c|}
\hline Factor & Odds ratio & $95 \% \mathrm{Cl}$ & $P$ \\
\hline \multicolumn{4}{|l|}{ Health behavior \& cognition } \\
\hline \multicolumn{4}{|l|}{ Current alcohol drinker } \\
\hline Yes & 0.787 & & 0.106 \\
\hline No (ref) & 1.000 & & \\
\hline \multicolumn{4}{|l|}{ Current smoker } \\
\hline $\mathrm{No}^{*}$ & 0.892 & & 0.383 \\
\hline Yes (ref) & 1.000 & & \\
\hline \multicolumn{4}{|c|}{ Subjective cognition of body shape } \\
\hline Normal $^{*}$ & 0.123 & & 0.000 \\
\hline Obese* & 0.024 & & 0.000 \\
\hline Underweight (ref) & 1.000 & & \\
\hline \multicolumn{4}{|l|}{ Stress } \\
\hline Nearly none* & 0.631 & & 0.005 \\
\hline A little & 0.859 & & 0.179 \\
\hline A lot (ref) & 1.000 & & \\
\hline \multicolumn{4}{|l|}{ Sitting hours (per day) } \\
\hline Fewer than 7 hours & 1.114 & & 0.450 \\
\hline Between 7 and 12 hours & 1.121 & & 0.393 \\
\hline 12 hours or more (ref) & 1.000 & & \\
\hline \multicolumn{4}{|c|}{ Amount and type of food eaten } \\
\hline Sufficient but not varied & 1.046 & & 0.275 \\
\hline Insufficient & 0.736 & & 0.228 \\
\hline Sufficient and varied (ref) & 1.000 & & \\
\hline
\end{tabular}

and female adults of all ages. Women, urban residents, and middle school graduates made more weight reduction efforts than other Korean adults. Additionally, individuals aged 61 to 80, those with a high income level, and those who were middle school graduates or higher made more weight control efforts than other Korean adults. Weight reduction efforts were associated with a perceived subjective health status of fair or healthy, hypertension, normal BMI, or being obese. Moreover, adults who were alcohol drinkers, nonsmokers, or who perceived themselves as being obese practiced more weight reducing efforts than other Korean adults.

Past research has shown that unmarried women typically engage more in weight-loss practices than married women, ${ }^{9}$ but we did not find a statistically significant difference in weight loss efforts between married and unmarried people. It might be that individuals in their 70s and 80 s practiced more weight maintaining efforts to prevent and treat chronic diseases. Similar to O'Brien et al., ${ }^{6}$ we found that women engaged in more weight control practices than men, and those attempting weight loss were overwhelmingly wom- 
en. However, O'Brien et al. ${ }^{6}$ also note that psychosocial factors can motivate attempts to adjust weight and that appearance disorders and mood conditions are additional reasons that individuals attempt weight control.

Previous studies showed that women people who perceived themselves to be obese, regardless of their actual BMI, engaged in more weight loss practices than those who did not consider themselves to be obese. ${ }^{9,12}$ Consistent with these previous studies, we demonstrated that individuals who we considered obese based on $\mathrm{BMI}$ and those that considered themselves to be obese regardless of BMI engaged in more weight loss practices than non-obese people. Thus both subjective obesity and actual obesity influence weight control practices. A previous study of Community Health Survey data reported that weight control practices differed according to subjective health levels; that is, those who had attempted to lose weight had adequate health levels. ${ }^{9}$ We also found that people who thought they were in good health made significantly more weight loss efforts than those who considered their health to be poor.

Weight control practices, especially weight loss practices, are very effective at reducing diabetes and cardiovascular disease. ${ }^{13-20}$ According to the DECODE study group, ${ }^{13}$ an increase in BMI among Finnish people was accompanied by an increase in type 2 diabetes, and weight control was needed to reduce type 2 diabetes. Furthermore, the Nurses' Health Study showed that the risk of diabetes was the lowest in patients with a BMI $<21 \mathrm{~kg} / \mathrm{m}^{2}$, and the higher the obesity rate, the higher the diabetes risk. ${ }^{14,15}$ Moreover, people with diabetes were less successful at long-term weight control, likely due to differences in metabolism compared to those without diabetes. However, diabetics need continuous monitoring of weight. ${ }^{16}$ The Finish Diabetes Prevention Study showed that weight control through lifestyle counseling prevented or at least delayed type 2 diabetes for those with a high risk of type 2 diabetes. ${ }^{17}$ Weight loss through lifestyle counseling was a strong indicator of a decrease in diabetes risk throughout the study period, and diabetes risk was halved in the group with a weight loss of $4 \%-6 \%$ compared to the group without weight change. Furthermore, the study "Look AHEAD (Action for Health in Diabetes)" in the U.S. reported that the risk of cardiovascular disease and medication use was significantly reduced in people who lost weight (study group) ${ }^{18,19}$
Weight control, especially by exercising, can improve insulin sensitivity in healthy people and in those with cardiovascular diseases. Intentional weight control affects blood pressure, inflammation, vascular function, and cardio-metabolic risk factors such as lipid levels. ${ }^{20}$ Moreover, deliberate weight control can easily modify risk factors for cardiovascular disease onset in overweight individuals. We found that those individuals with high blood pressure made more weight loss efforts than those with other health conditions. More active weight loss efforts are required in people with diabetes, emphasizing the need for education and support among this subgroup of the population.

In an American study, more weight loss efforts were seen in adults who had hypertension or diabetes than those without. ${ }^{21}$ In our study, after controlling for related variables, only individuals with hypertension engaged in more weight loss practices than those with other health conditions. Therefore, the health benefits of controlling hypertension and diabetes through weight control need to be further emphasized in Korea. Furthermore, patients can set appropriate weight-loss health goals and improve their health if they are educated about developing effective weight-loss strategies.

Having a sedentary lifestyle during the day versus a more active lifestyle was not associated with weight loss practices, contrary to the results reported by Kim?: weight-loss behaviors may be less associated with sedentary time during the week, but may be more associated with exercise. In this sense, it would be good if variables for exercise were also taken into account in future studies. In general, drinking and smoking are known to have a significant negative impact on health. However, a previous study that examined the relationship between smoking and weight loss practices ${ }^{9}$ showed no difference in weight loss according to smoking status. By contrast, a study by Tamim et al. ${ }^{22}$ targeting college students showed a significant difference in weight loss depending on whether or not the students smoked. Similarly, we found that non-smokers had more weight loss or maintenance practices. Additionally, people who drank had more weight loss practices in our study. Apart from weight loss practices, people need to improve their health through smoking cessation and moderation in drinking. This is because drinking and smoking are unhealthy behaviors related to chronic disease.

This study used recent data to identify weight control factors for 
Korean adults in general, instead of a specific population group. Women, individuals over 51 years of age, city dwellers, those with a middle school or higher graduation level, individuals who perceived themselves to be healthy or in fair health, people with high blood pressure, those with a normal or obese BMI, non-smokers, and those who drank alcohol made more weight loss efforts than other Korean adults. These results indicate that it is necessary to monitor and address the lack of weight loss efforts in rural residents and that there is a need for weight loss counseling and support for people with high blood pressure. Additionally, further research is needed to identify whether alcohol drinkers' weight loss efforts are an effort to compensate for the adverse health practices of drinking. If so, community efforts to reduce the health risks posed by drinking are needed.

This study had several limitations. As this was a cross-sectional study, a causal relationship between weight control practices and related factors could not be inferred. Because of the possibility of reverse causality, i.e., more efforts to reduce weight in individuals with hypertension, the observed association between individuals with hypertension and weight loss efforts may be, at best, a mixture of causal effects in both directions. In fact, it could be that people with diabetes may need more education on weight control because they made less effort to lose weight than individuals without diabetes according to our study findings. Causal relationships can only be determined by long-term longitudinal studies, and the results here should be interpreted with a focus on relevance. Furthermore, although some people in the study were trying to maintain weight or increase their weight, the majority of individuals made efforts to reduce their weight. Similarly, most previous studies focused on weight loss, so factors associated with weight maintenance and weight gain have rarely been explored. These factors need to be investigated further in future studies.

Weight control is a positive practice for people with obesity, hypertension, or diabetes. However, those who believe that they are obese even when their weight is normal may require education regarding weight control and health and further observation. Furthermore, despite the high rates of obesity among individuals living in rural areas, these individuals made fewer weight control efforts than those living in urban areas. Thus, more detailed investigations are needed to explore the link between disease and weight control.
Further studies should consider various related causes and may need to consider community-wide efforts to support people who need weight control.

\section{CONFLICTS OF INTEREST}

The authors declare no conflict of interest.

\section{AUTHOR CONTRIBUTIONS}

Study concept and design: HYL; acquisition of data: all authors; analysis and interpretation of data: all authors; drafting of the manuscript: all authors; critical revision of the manuscript: all authors; study supervision: all authors.

\section{REFERENCES}

1. Kim YO. Weight control behaviors among Korean adults: association with dietary intake. J Korean Soc Food Sci Nutr 2002;31:1018-25.

2. Chaung SK. Weight control practices and body image of female college students. J Korean Soc Health Edu Promot 2001; 18:163-75.

3. Neumark-Sztainer D, Wall M, Story M, Standish AR. Dieting and unhealthy weight control behaviors during adolescence: associations with 10-year changes in body mass index. J Adolesc Health 2012;50:80-6.

4. Smith DW, McFall SL. The relationship of diet and exercise for weight control and the quality of life gap associated with diabetes. J Psychosom Res 2005;59:385-92.

5. Pan HJ, Cole BM, Geliebter A. The benefits of body weight loss on health-related quality of life. J Chin Med Assoc 2011;74:169-75.

6. O'Brien K, Venn BJ, Perry T, Green TJ, Aitken W, Bradshaw A, et al. Reasons for wanting to lose weight: different strokes for different folks. Eat Behav 2007;8:132-5.

7. Lee WO, Hwang YJ. A study on high-school girls' obesity, attitudes towards body and weight control. Korea Institute Youth Facility Environ 2009;7:3-11.

8. Oh DN, Kim EM, Kim SH. Weight control behaviors and 
correlates in Korean adolescents. J Korea Contents Assoc 2013;13:218-28.

9. Kim JH. Associations between weight control behavior and health behaviors in Korean young women: 2013 community health survey. J Korea Academia-Industrial Coop Soc 2015; 16:4734-44.

10. Yang SK, Ha YM, Jung MR. Convergence relationships among body-esteem, obesity stress, self-esteem, weight control behavior in high school girls. J Digit Converg 2017;15:237-45.

11. Ministry of Health and Welfare; Korea Centers for Disease Control and Prevention. Fifth Korea National Health and Nutrition Examination Survey (KNHANES) in 2016-2018. Sejong: Korea Centers for Disease Control and Prevention; 2018.

12. Yang HY, Byeon YS. Weight control behavior in women college students and factors influencing behavior. J Korean Acad Fundam Nurs 2012;19:190-200.

13. DECODE Study Group. Age- and sex-specific prevalences of diabetes and impaired glucose regulation in 13 European cohorts. Diabetes Care 2003;26:61-9.

14. Carey VJ, Walters EE, Colditz GA, Solomon CG, Willett $\mathrm{WC}$, Rosner BA, et al. Body fat distribution and risk of noninsulin-dependent diabetes mellitus in women: the nurses' health study. Am J Epidemiol 1997;145:614-9.

15. Carey VJ, Walters EE, Colditz GA, Solomon CG, Willett WC, Rosner BA, et al. Body fat distribution and risk of non-insulindependent diabetes mellitus in women: the nurses' health study. Am J Epidemiol 1997;145:614-9.

16. Wing RR, Goldstein MG, Acton KJ, Birch LL, Jakicic JM, Sallis JF Jr, et al. Behavioral science research in diabetes: lifestyle changes related to obesity, eating behavior, and physical activity. Diabetes Care 2001;24:117-23.

17. Lindström J, Peltonen M, Tuomilehto J. Lifestyle strategies for weight control: experience from the Finnish Diabetes Prevention Study. Proc Nutr Soc 2005;64:81-8.

18. Ryan DH, Espeland MA, Foster GD, Haffner SM, Hubbard VS, Johnson KC, et al. Look AHEAD (Action for Health in Diabetes): design and methods for a clinical trial of weight loss for the prevention of cardiovascular disease in type 2 diabetes. Control Clin Trials 2003;24:610-28.

19. Look AHEAD Research Group, Pi-Sunyer X, Blackburn G, Brancati FL, Bray GA, Bright R, et al. Reduction in weight and cardiovascular disease risk factors in individuals with type 2 diabetes: one-year results of the look AHEAD trial. Diabetes Care 2007;30:1374-83.

20. Ades PA, Savage PD. Potential benefits of weight loss in coronary heart disease. Prog Cardiovasc Dis 2014;56:448-56.

21. Zhao G, Ford ES, Li C, Mokdad AH. Weight control behaviors in overweight/obese U.S. adults with diagnosed hypertension and diabetes. Cardiovasc Diabetol 2009;8:13.

22. Tamim H, Tamim R, Almawi W, Rahi A, Shamseddeen W, Ghazi A, et al. Risky weight control among university students. Int J Eat Disord 2006;39:80-3. 